\title{
Flexible CubeSat-Based System for Data Broadcasting
}

\author{
S. Jayousi, S. Morosi, University of Florence, Italy \\ L. S. Ronga, Leonardo, Security and Information Systems, Campi Bisenzio, Italy \\ $E$. Del Re, University of Florence, Italy \\ A. Fanfani, L. Rossettini, D-Orbit srl, Milano, Italy
}

\section{INTRODUCTION}

\section{BACKGROUND AND MDTIVATION}

Effective answers to the major problems in society can be achieved by resorting to the integration of telecommunication platforms, heterogeneous systems of localization, and sensing capabilities and intelligent objects [1]. Particularly, the pursuit of long term technological solutions has to be made with the support of a revolutionary multidisciplinary approach, especially in the areas of transport, smart environments, quality of life, security, etc.

As a result, new and more intelligent services will become available, allowing the realization of smart contexts for what concerns home, building, cities, work-places, and implementing new concepts as ambient intelligence, ambient assistant living, ubiquitous computing, context awareness, and many more. This pervasive communication ecosystem will solve many, if not most, problems in our life, by increasing security and safety perception and improving interaction and conditions of activities; information and communications technologies will be a pillar of this society evolution by providing a complex net of information exchanges and exploiting the big data that are involved in the procedures [2].

Moreover, the satellite segment will contribute significantly to the evolution of the services by means of the synergistic use of communication, positioning, and monitoring techniques: this objective will be realized by means of meshed heterogeneous architectures [2] that can afford remarkable systems and networks performance. At the same time, some of the envisaged future services such as the global tracking, monitoring, and maintaining of some specific assets or contexts don't mandatorily request the implementation of classic wireless or satellite communication systems

Authors' current addresses: S. Jayousi, S. Morosi, E. Del Re, University of Florence, Information Engineering Department, Via di S. Marta, 3 Florence, I-50139 Italy; L. S. Ronga, Leonardo-Security and Information Systems, Via Albert Einstein 35, Campi Bisenzio, 50013, Italy; A. Fanfani, L. Rossettini, D-Orbit srl, Via Giuseppe Mazzini 2, 20123 Milano (MI), Italy.

Manuscript received June 7, 2017, revised November 5, 2017, December 19, 2017, and ready for publication December 23, 2017.

Review handled by L. Ligthart. 0885/8985/18/\$26.00 @ 2018 IEEE which are subject to coverage and cost limitations; conversely, the uprising Machine-to-Machine (M2M) and Internet of Things paradigms seem to be more appropriate in such scenarios where a global packet connectivity has to be afforded in order to permit the exchange of information between the network and the ultimate users but the requirements in terms of data-rate and latency are not stringent [3], [4].

On the other hand, this strategy can be successful if some operating conditions and key factors can be granted; particularly, the following conditions are compelling:

- this strategy has to be general, i.e., a very large set of future services has to rely on it, even if their requirements are rather diversified and even divergent;

- the communications, both within the system and between the system and the external world, will have to be highly efficient and to deliver all the necessary information in due time, where needed, with required quality of service (QoS) and energy consumption.

Among the systems which allow the achievement of both of these goals, the new class of "SmallSats" also affords the reduction of the implementation costs and the increase of system availability because of their recent exponential growth [5], [6], [7]. As a result, the exploitation of SmallSats solutions, such as the CubeSat, as a basic element of the problem-solving infrastructures has been envisaged.

Thanks to CubeSat standardization, low cost of commercial off-the-shelf (COTS) electronics and new launch opportunities, constellations of SmallSats that are able to provide a realistic worldwide coverage represent a promising solution for the provision of different communication services. Some examples are: the AAUSAT3 mission for the validation of an Software Defined Radio (SDR) automatic identification system (AIS) signals receiver for CubeSat [8]; the Outernet projects for broadcasting free content retrieved from the web through satellites [9]; Astrocast project for offering global M2M services as remote monitoring, geolocalization, intelligent data collection, and predictive maintenance [10].

Moreover, the heterogeneity of the potential application scenarios, and therefore the flexibility needed on the receiver side, makes the SDR technology [11] suitable for the quick adaptation of the receiver to different physical layers, maximizing the opportunity to disseminate multiple information messages all over the world [12]. 


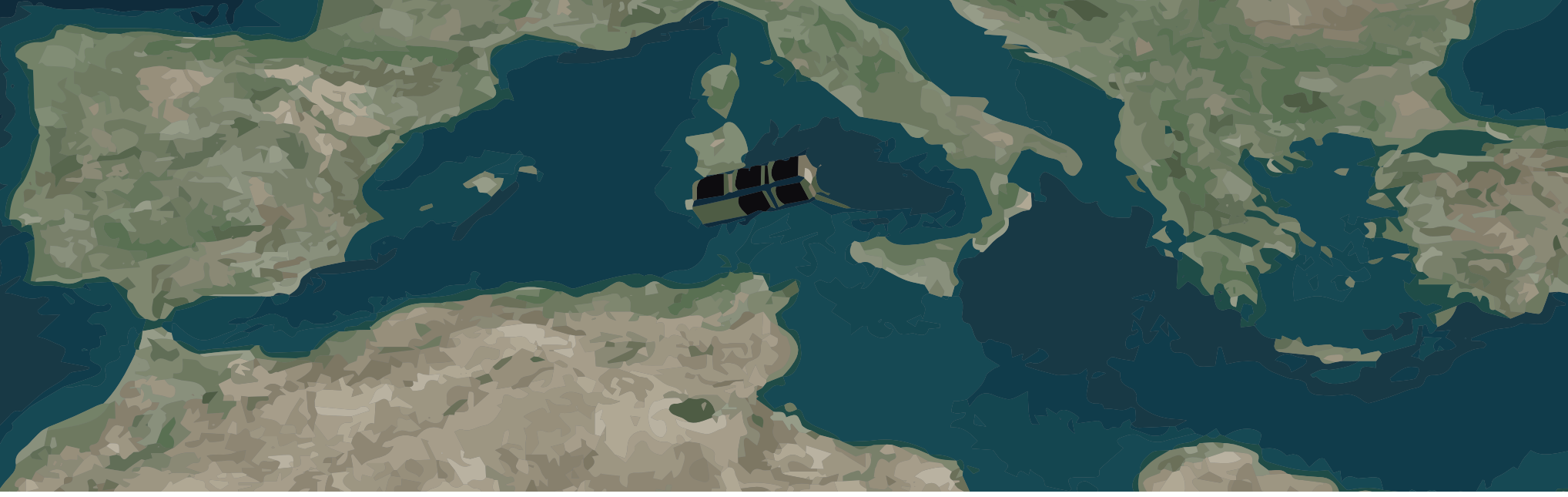

\section{QUR CONTRIBUTION}

The objective of this article is to propose a flexible system architecture for data broadcasting based on the adoption of: i) data gathering, processing, and formatting techniques and protocols; ii) a CubeSat for messages broadcasting; iii) SDR technology for messages reception; iv) data exchange mechanisms for information sharing among the system entities. Its applicability to different contexts, ranging from that of e-health and well-being to info-mobility, is highlighted. Particular attention is devoted to the analysis of an emergency scenario by providing details on an experimental demonstration of a designed and developed alert messaging system that has been validated in the framework of the Deorbit Satellite (D-SAT) Mission. The adoption of satellite technology in the provision of broadcast services and, in particular in the specific context of Emergency Communication, is further justified by several benefits, which range from technical to management aspects. One of the key factor is represented by the uniform (also in terms of QoS) and wide coverage the satellite provides. This allows to reach large areas, including those area characterized by the unavailability or absence of an adequate terrestrial network connection. Moreover, low Earth orbit (LEO) satellites enable the access

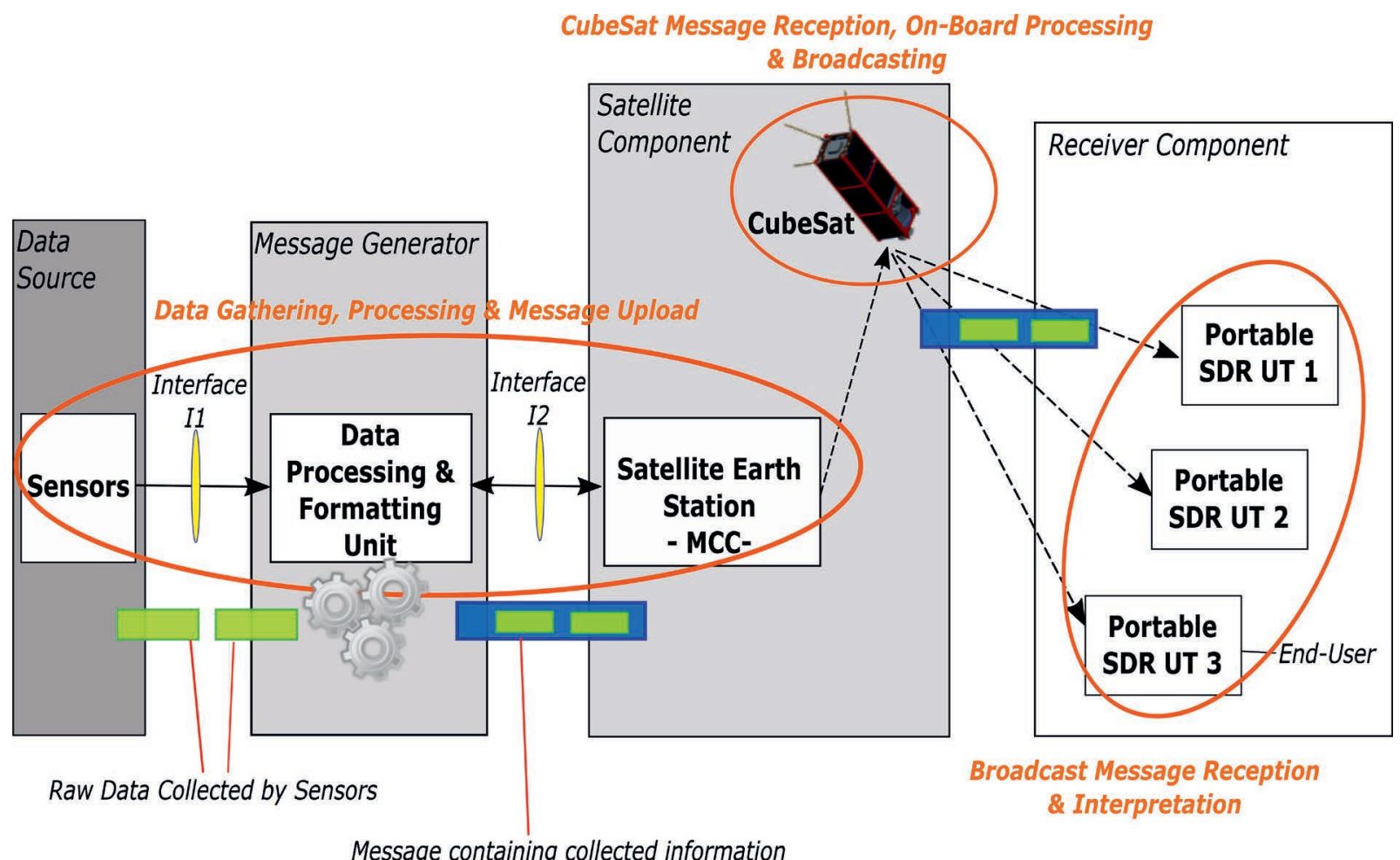

Legend:

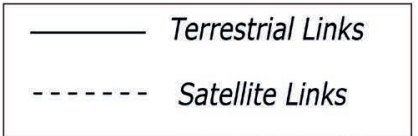

Figure 1.

Flexible CubeSat-based system architecture for data broadcasting. 
to remote areas such as North and South poles that are not covered by geosynchronous satellite's footprints. In addition, the resilience of satellite technology with respect to terrestrial infrastructure is the value proposition of the proposed solution. The adoption of CubeSat and therefore of CubeSat constellations represents a good opportunity and an innovative solution for the delivery of broadcast messages in different application contexts. This is enhanced by the CubeSat standardization, low cost of COTS electronics, and new launch opportunities. Finally, it is worth underlining that, to the best of our knowledge, the proposed CubeSat-based system and the D-SAT mission are the first application of these concepts and strategies to the data broadcasting in emergency scenarios: the results of the mission demonstrate that this original approach is effective in terms of performance, coverage, and costs [13], [14]; moreover the specific context of emergency and the need of delivering alert messages to the population over a large area led to the adoption of satellite technology that may represent the only solution to reach the intended audience in time.

\section{SYSTEM MODEL}

The high-level architecture of the proposed flexible CubeSatbased system for data broadcasting is depicted in Figure 1. To better analyse the functioning of the whole transmission and reception chain, following the information flow from the source (left side) to the destination (right side), three macrofunctional components are identified and detailed. These are:

- Data gathering, processing, and message upload.

- CubeSat message reception, on-board processing, and broadcasting.

- Broadcast message reception and interpretation.

\section{DATA GATHERING, PROCESSING, AND MESSAGE UPLDAD}

The Data Source can be represented by sensors distributed over a monitored area or by other sensing/monitoring systems. It is responsible for gathering raw data and transmitting them to a collector for a preliminary processing.

The collector, named the Data Processing and Formatting Unit (Figure 1), is in charge of processing the raw data coming from the Data Source and generating an opportunely formatted message that includes all the needed information extracted from the received data. Different transport protocols and formats can be implemented by the Data Processing and Formatting Unit, depending on the specific application context. An example is reported later in this article.

The message is then received by the Satellite Earth StationMission Control Center (MCC), which proceeds with the upload of it to the CubeSat for the consecutive broadcasting.

As highlighted in Figure 1, the interfaces between the Data Source and the Data Processing and Formatting Unit (Interface I1) and the one between the Data Processing and Formatting Unit and the Satellite MCC need to be defined to allow the proper information exchange among the involved entities. Details on a possible implementation of such interfaces are reported later.
CuBESAt MESSAGE RECEPTION, QN-BDARD PRDCESSING

\section{AND BRDADCASTING}

The space component is represented by a CubeSat, whose main functions are: i) the reception of the messages uploaded by the Satellite $M C C$; ii) the processing of the received messages and consecutive storage of them, if needed; iii) the messages broadcasting.

Without losing in generality, in this section, the implementation of a CubeSat (named D-SAT) is described. D-SAT, that is shown in Figure 2, despite of its compact dimension [ $30 \times 10 \times$ 10) $\mathrm{cm}^{3}$ ] and its mass lower than $4.5 \mathrm{~kg}$, includes all the typical satellite subsystems. The On-Board Computer (OBC) comprises Attitude and Orbit Control System (AOCS) sensors (magnetometers, sun sensors, gyroscope) and the drivers for AOCS magnetorquers. The core of the OBC is a flight proven High-performance ARM7 Central Processing Unit.

The OBC has an on-board timer which is synchronized with Coordinated Universal Time by means of a Global Positioning System receiver within the satellite. The communication subsystem consists of an Ultra High Frequency (UHF) radio module and a turnstile antenna. The Electric Power System includes batteries and solar arrays and the D-Orbit Decommissioning device that has been conceived to dispose the satellite.

In details, the communication subsystem includes an omnidirectional antenna and an UHF transceiver that uses a Gaussian Minimum Shift Keying (GMSK) modulation over Frequency Modulation signal at a baud rate of 4,800 bps. The bandwidth of the signal is $14.6 \mathrm{kHz}$. For further information, the CubeSat GMSK bandwidth is $4,800 \mathrm{~Hz}$ and frequency deviation is $2.5 \mathrm{kHz}$. The data are coded with a Reed-Solomon $(223,255)$ coding and randomized according to the Consultative Committee for Space Data Systems (CCSDS) standard to reduce the probability of error and therefore increasing the reliability of the communication link. The choice of UHF band is the result of a trade-off between different bands: very high frequency (VHF), UHF, L-BAND, SBAND, and X-BAND. Technical and Programmatic criteria such as Complexity of on-board and ground radio equipment, antenna dimension, antenna gain, path-loss, atmospheric attenuation, Doppler effect, radio frequency license, and development cost were

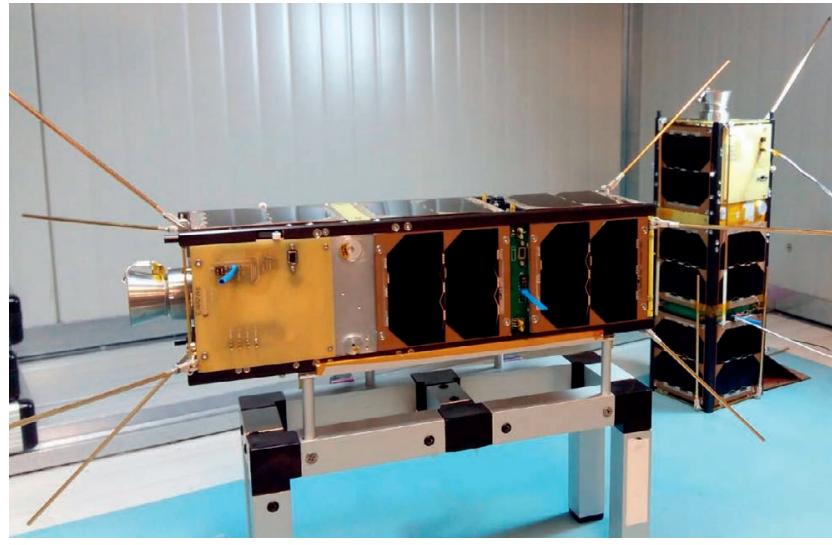

Figure 2.

D-SAT flight and qualification models in D-Orbit clean room facilitycourtesy of D-Orbit. 
Table 1.

\begin{tabular}{|c|c|c|c|c|}
\hline CubeSat-MCC Link Budget & Down-Link & Up-Link & CubeSat-SDR UT Link Budget & Downlink \\
\hline Satellite Range & $909.5 \mathrm{~km}$ & $909.5 \mathrm{~km}$ & Satellite Range & $909.5 \mathrm{~km}$ \\
\hline Elevation Angle & $30 \mathrm{deg}$ & $30 \mathrm{deg}$ & Elevation Angle & $30 \mathrm{deg}$ \\
\hline Frequency & $437.5 \mathrm{MHz}$ & $437.5 \mathrm{MHz}$ & Frequency & $437.5 \mathrm{MHz}$ \\
\hline Transmission Rate & 4,800 bps & 4,800 bps & Transmission Rate & 4,800 bps \\
\hline \multicolumn{3}{|l|}{ Transmitter } & \multicolumn{2}{|l|}{ Transmitter } \\
\hline Transmitted Power & $1 \mathrm{~W}$ & $10 \mathrm{~W}$ & Transmitted Power & $1 \mathrm{~W}$ \\
\hline Antenna Gain & $0.4 \mathrm{dBi}$ & $17.6 \mathrm{dBi}$ & Antenna Gain & $0.4 \mathrm{dBi}$ \\
\hline Line Losses & $-1.7 \mathrm{~dB}$ & $-3.9 \mathrm{~dB}$ & Line Losses & $-1.7 \mathrm{~dB}$ \\
\hline Pointing Losses & $-3 \mathrm{~dB}$ & $-3 d B$ & Pointing Losses & $-3 d B$ \\
\hline \multicolumn{3}{|l|}{ Channel } & \multicolumn{2}{|l|}{ Channel } \\
\hline Path-Loss & $-144,4 \mathrm{~dB}$ & $-144,4 \mathrm{~dB}$ & Path-Loss & $-144,4 \mathrm{~dB}$ \\
\hline Polarization Loss & $-3 \mathrm{~dB}$ & $-3 d B$ & Polarization Loss & $0 \mathrm{~dB}$ \\
\hline Atmospheric Losses & $-1.8 \mathrm{~dB}$ & $-1.8 \mathrm{~dB}$ & Atmospheric Losses & $-1.8 \mathrm{~dB}$ \\
\hline \multicolumn{3}{|l|}{ Receiver } & \multicolumn{2}{|l|}{ Receiver } \\
\hline Antenna Gain & $17.6 \mathrm{dBi}$ & $0.4 \mathrm{dBi}$ & Antenna Gain & $5 \mathrm{dBi}$ \\
\hline HBPW & $21 \mathrm{deg}$ & $180 \mathrm{deg}$ & HBPW & $80 \mathrm{deg}$ \\
\hline Figure of Merit, $(\mathrm{G} / \mathrm{T})$ & $-10.4 \mathrm{~dB} / \mathrm{K}$ & $-25.2 \mathrm{~dB} / \mathrm{K}$ & Figure of Merit, (G/T) & $-10.3 \mathrm{~dB} / \mathrm{K}$ \\
\hline \multicolumn{3}{|l|}{ Results } & \multicolumn{2}{|l|}{ Results } \\
\hline Received Isotropic Power & $-113.5 \mathrm{dBm}$ & $-97.9 \mathrm{dBm}$ & Received Isotropic Power & $-113.5 \mathrm{dBm}$ \\
\hline$E_{b} / N_{0}$ & $28.3 \mathrm{~dB}$ & $37.3 \mathrm{~dB}$ & $E_{b} / N_{0}$ & $18 \mathrm{~dB}$ \\
\hline Required $E_{b} / N_{0} @ \mathrm{BER}=10^{-5}$ & $13.5 \mathrm{~dB}$ & $13.5 \mathrm{~dB}$ & Required $E_{b} / N_{0} @ \mathrm{BER}=10^{-5}$ & $13.5 \mathrm{~dB}$ \\
\hline Margin $E_{b} / N_{0}$ & $14.8 \mathrm{~dB}$ & $23.7 \mathrm{~dB}$ & Margin $E_{b} / N_{0}$ & $4.5 \mathrm{~dB}$ \\
\hline
\end{tabular}

selected and weighted. A small stilo UHF antenna ensures a quasiomnidirecitonal radiation pattern, unlike patch antennas that need an accurate pointing. UHF Yagi antenna on-ground has quite-wide Half Power Beam Width that simplifies satellite tracking without excessively harming the antenna gain. The Doppler shift, the pathloss, and the atmospheric attenuation in UHF are lower with respect to the higher bands and finally the electronic components working in UHF are cheaper. At the time of definition of the satellite components, the only radio with high reliability and extensive flight heritage featured a GMSK modem. Even though the choice of GMSK modulation was imposed by the availability of COTS on-board radio for CubeSats, nonetheless the GMSK solution resulted the best for our application due to the high reliability and the possibility to use an incoherent demodulator.

The communication subsystem main functions are: i) the transmission of a beacon signal (a continuous wave signal frequencymodulated) as tracking signal; ii) the transmission of the Telemetry signals and the reception of Telecommand signals; iii) the reception of the information messages from the MCC and the broadcast of them. The link frequency value is $437.505 \mathrm{MHz}$ and is coordinated by the International Amateur Radio Union and assigned by the In- ternational Telecommunication Union (ITU). As for the Transport level, the NanoCom U482C uses CubeSat Space Protocol, a small network-layer delivery protocol based on a 32-bit header.

The satellite uses a redundant architecture so the on-board communication is also duplicated. In order to minimize antennas coupling, the master unit's antenna uses RCP and the back-up unit's antenna uses LCP.

The telemetry and telecommand link between the CubeSat and the MCC was designed according to the link budget reported in Table 1: particularly, the values of the Figures of Merit and of the atmospheric losses has been both derived from literature [16], whereas the value for Line Losses and Required $E_{b} / N_{0}$ has been obtained from the technical datasheets of the components; this is the reason why the value of the required $E_{b} / N_{0}$ has been taken equal to $13.5 \mathrm{db}$. Finally, the experimental nature of the SatAlert Mission has pushed us to a precautionary approach against all the possible sources of failures or performance impairments and, therefore, we have decided to keep the margin values higher than strictly necessary; on the other hand, it is important to highlight that their reduction would have not brought any significant benefit to both space and ground segments. As a matter of fact, a huge attention has been 


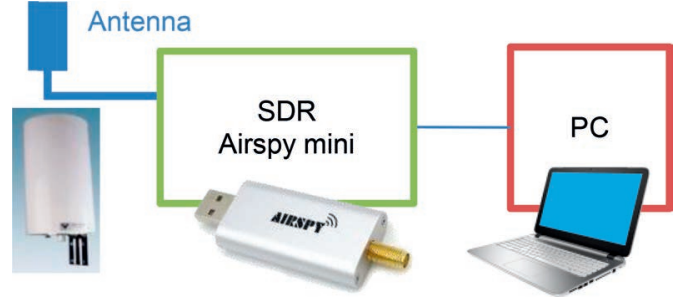

Figure 3 .

Block diagram of the portable SDR user terminal.

devoted to the budget link definition since it allows to compare the envisaged figure of merits with the ones that are reported for similar SmallSats missions.

\section{BROADCAST MESSAGE RECEPTION AND INTERPRETATION}

In order to meet different application contexts, which may be characterized by limited availability of resources and infrastructure, a low-cost portable terminal is considered as a receiver component. Its main functions are the reception and the decoding of the broadcast messages. The low complexity and flexibility of the proposed solution are achieved by the adoption of the SDR technology. The block diagram of the designed and implemented satellite SDR portable user terminal (UT) is depicted in Figure 3. It includes three main elements: a Circular Polarized Quadrifilar Helix Antenna, an Airspy Mini SDR radio, and a personal computer (PC) configured with GNU Radio software.

The D-SAT operative scenario and the impossibility of using a rotator mechanism to point the satellite lead to the adoption of a Quadrifilar Helix Antenna instead of a directive one. The Helix Antenna ensures a semispherical radiation pattern characterized by a $5 \mathrm{~dB}$ maximum antenna gain and half power beam-width up to \pm 80 deg. and circular polarization matched with D-SAT's antenna. Despite the limited gain, the $E_{b} / N_{0}$ value is still high enough for correctly decoding the signal as reported in Table 1.

The selected SDR-platform is the AirSpy Mini SDR, supported by a GNU Radio implementation on a computer. The main performance of the AirSpy Mini SDR are: a $3.5 \mathrm{~dB}$ Noise Figure in UHF frequency band, a $35 \mathrm{dBm}$ IIP3 RF front end amplifier, a 12-bit Analog-to-Digital Converter with a Dynamic Range up to $80 \mathrm{~dB}$; a 0.5 ppm high precision, low phase noise clock.

The PC hosts Linux operative system running GNU Radio [15], CubeSat Space Protocol framework, and an User Interface application that is written in $\mathrm{C}$ language and provides the satellite messages to the users. Modulation and demodulation, error detection and correction, as well as clock synchronization and Doppler compensation are performed by GNU Radio [17], [18].

\section{ENABLING CDMMUNICATION AMONG HETERDGENEQUS COMPONENTS: WEB SERVICE PARADIGM}

A set of Web Services (WSs) [19] can be defined to automate the interactions among the Data Source, the Data Processing and Formatting Unit, and the Satellite MCC. WSs enable an automatic exchange and processing of information, allowing the involved en- tities to interface one to each other. In fact, WS architectures, data serialization methods, Application Programming Interfaces (APIs) are the key elements for the integration of several heterogeneous components. A possible solution for message data exchanging can be represented by the adoption of JavaScript Object Notation (JSON) [20] as data serialization method. This allows to simplify the development, integration and debugging.

In order to simplify the development, integration, and debugging, a possible solution for message data exchanging relies on JSON [20] data serialization. Referring to Figure 1, the APIs for Interface I1 are oriented to the message generation and enable the transmission of the raw data to the Data Processing and Formatting Unit for the consecutive processing and formatting operations. While APIs for Interface I2 are oriented to the message provision and allow the Satellite MCC to get the messages that have to be uploaded to the CubeSat.

\section{POTENTIAL APPLICATION CONTEXTS}

As described in the Introduction, the synergistic use of communication, positioning, and monitoring techniques will contribute to determine effective solutions for major social problems while the exploitation of "SmallSats" can significantly cut the implementation costs and improve the service availability.

The defined architecture can be adopted for the provision of multiple services based on the distribution of messages over a large area (e.g. alert services, traffic/transport information, advertising, etc.). Today many applications rely on data gathering, processing, and communication and the proposed architecture, including the set of Web services that has been developed for interfacing the different entities and automate the message generation process, represents a baseline system that can be easily adopted to several application contexts. This highlights the flexibility of the proposed system at service level. In addition, the adoption of a portable Software Defined Network Receiver on end user side to decode the messages represents a low-cost solution to receive and interpret the broadcast messages directly from the satellite, contributing to the flexibility of the system at receiver level.

Particularly, in the following some of the contexts which could benefit from the adoption of the solutions that are based on the use of the CubeSat are identified and discussed:

- Health and wellbeing. Beyond being one of the most serious environmental issues, the atmospheric pollution has become one of the main causes of the degradation of the quality of life, with increased adverse effects on human health [21]; particularly, the ozone $O_{3}$ can generate several negative effects on human health due to its nature of reactive oxidant agent [22]. In this context, the proposed system could provide alert messages about the presence of Ozone in a given area to the people that is going to cross the involved area.

- Smart, green, and integrated transports. The fast response of the SmallSats systems is indeed useful in tracking traffic jams and provides assistance to the drivers by suggesting alternative paths and shorter routes. 
- Security and emergency. Solutions that are characterized by the integration of CubeSat can be beneficial in the four different phases of the management of emergency situations [23] in order to deliver information both to the stakeholders and to the population: these capabilities are absolutely crucial both in catastrophic events and in terroristic attacks.

It is worth stressing that the considered services are mainly broadcast ones; even if the provision of such services is now being conceived for the portable UT, as described in the previous section, future diffusion of the considered broadcast services could open the way to solutions tailored for hand-held devices (e.g. a Smartphone).

\section{CASE STUDY: ALERT MESSAGES BRDADCASTING}

The objective of this section is to show the applicability of the proposed system to an Emergency scenario. Starting from the architecture that has been previously defined, an Alert Messaging System which is able to efficiently deliver alert messages over the incident area, has been designed and developed: the building blocks of the broadcast architecture and their integration have been introduced in [24], [25]. In the following subsections the implemented Alert Messaging System is described, providing an overview of the experimental activity carried out in the framework of the D-SAT Mission.

\section{EXPERIMENTAL ACTIVITY FRAMEWORK: THE D-SAT MISSION}

The proposed Alert Messaging System has been developed and implemented and the overall transmission-reception chain has been experimented in the framework of the D-SAT Mission (SatAlert Experiment) [26]. D-SAT spacecraft (previously described) has been designed and manufactured by D-Orbit and was launched by the Polar Satellite Launch Vehicle (PSLV) in LEO Sun-Synchronous Orbit at $500 \mathrm{~km}$ altitude in June 2017. The Mission main experimental activities have been the SatAlert Experiment and the Decommissioning Device in-orbit technology validation.

The objective of the SatAlert experiment was to demonstrate the feasibility of both the transmission over the space segment (LEO satellite) of alert messages encapsulated into MAMES (Multiple Alert Message Encapsulation over Satellite) protocol messages [27], [28] and their reception and decoding by a portable SDR UT. All the system components have been validated, including the on-board software that parses and broadcasts the MAMES messages [29].
On the other hand, the objective of the Decommissioning Device Experiment was to demonstrate the in-orbit capabilities of the D-Orbit Decommissioning device, that was responsible for the management and control of the satellite during the disposal phase. In fact, at the end of the mission, the Decommissioning Device safely completed the activation procedure and the Solid Rocket Motor was fired correctly.

\section{MAMES PROTOCOL MAIN FEATURES}

A comprehensive understanding of the different components of the developed Alert Messaging System requires a brief overview of the Multiple Alert Message Encapsulation over Satellite (MAMES) protocol main features [27], [28] and, in particular, of the MAMES frame structure. MAMES Message (Figure 4) consists of a set of headers and a payload, which comprises a concatenation of Alert Protocol Messages.

The MAMES Headers comprise: the Mandatory Header (MH), the Extension Headers (EHs), and the Alert Message Header $(\mathrm{AMH}) . \mathrm{MH}$ and EHs contain mandatory and optional/enhancing information, respectively, related to the entire MAMES Message. For each Alert Protocol Message (AM) contained in the payload, a corresponding AMH is defined, specifying the type, language, and length of the AM it refers to.

Focusing on the proposed Alert Messaging System, the definition of the priority and time validity information encoded in the MAMES Header fields is needed to introduce the MAMES Message processing performed on board of the CubeSat (D-SAT). In detail:

- the MAMES Transport Priority field of the MH manages the MAMES Frame priority with respect to other MAMES Frames at transport level;

- the MAMES Validity Start and End fields indicate the time instant when the MAMES Frame shall become valid and invalid, respectively.

\section{THE ALERT MESSAGING SYSTEM}

The high-level design of the Alert Messaging System developed for the proof of concept of alert messages generation, broadcast-

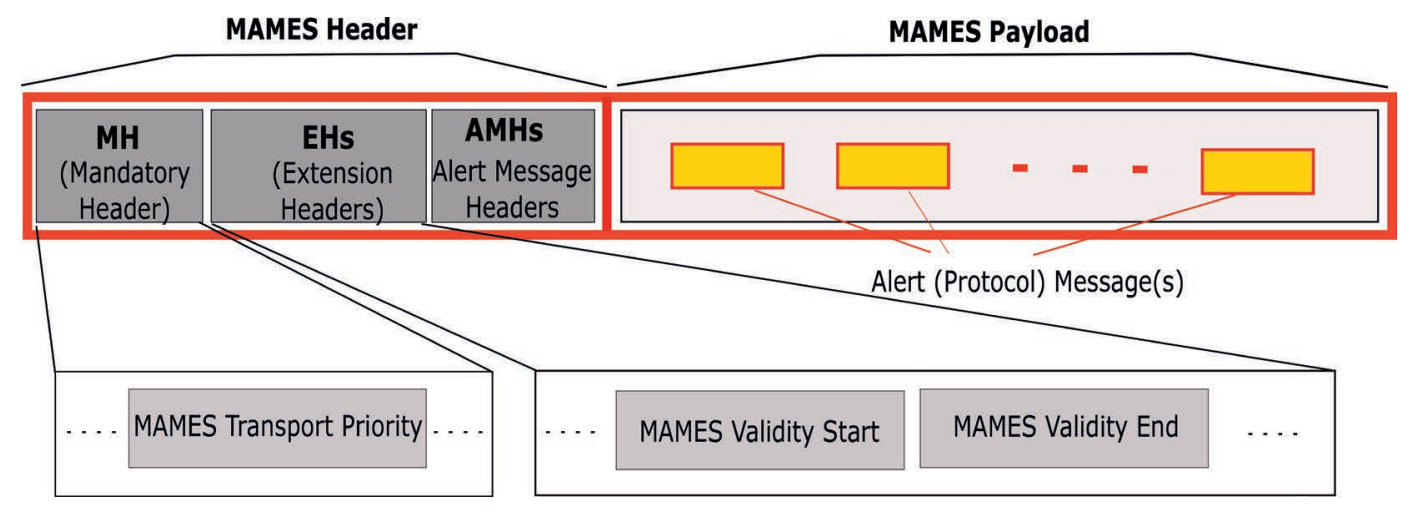

Figure 4.

MAMES message structure: Header and payload. 


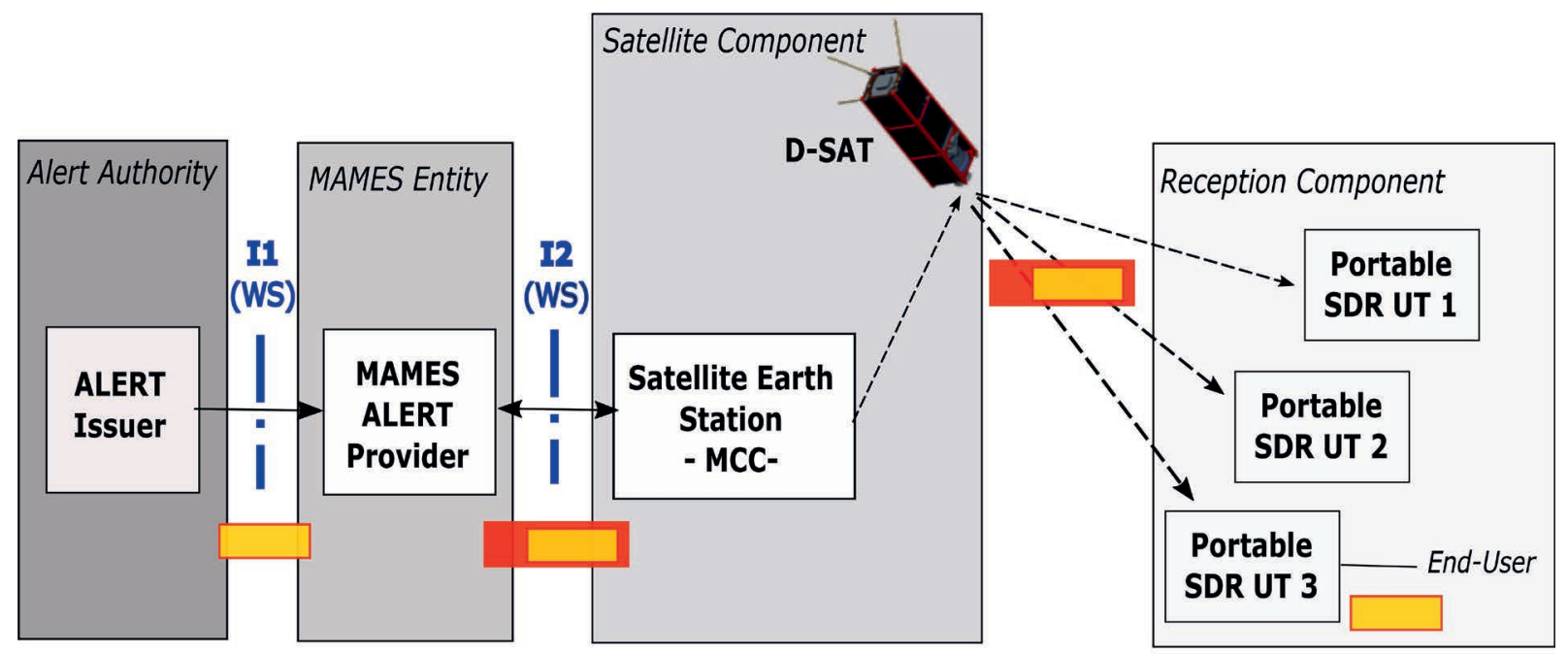

Legend:

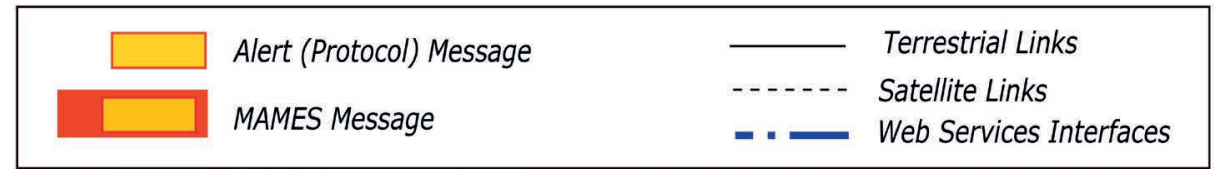

Figure 5.

Alert messages broadcasting system architecture.

ing, and reception is shown in Figure 5. From the left to the right side, the main components are:

- Alert Issuer, an alert authority responsible for generating the alert messages to be delivered.

- MAMES Alert Provider, the network entity responsible for encapsulating the alert messages into the MAMES ones as defined in [27].

- Satellite Component, a Satellite Mission Control Center (MCC) for the upload of MAMES messages and the satellite subsystems management and a 3-Unit CubeSat (D-SAT) in charge of processing and broadcasting the MAMES messages.

- Portable SDR User Terminal, a low complexity network entity for the reception of MAMES Messages and the decapsulation of alert ones.

The functioning of the overall system envisages the exchange of alert information among the involved entities.

The Alert Issuer provides the MAMES Alert Provider with the alert related information which includes both the AM (formatted according to the Alert Issuer most convenient format) and the additional information (such as validity, priority, event category, etc.) that is used for the MAMES Header definition. The MAMES Alert Provider encapsulates the received message into a MAMES Message and makes it available for the satellite communication network. The Satellite MCC periodically queries the MAMES Alert Provider for a new MAMES Message and uploads it to the D-SAT.

After MAMES message on-board processing (validity and priority checks), the D-SAT broadcasts the received message back to
Earth. The MAMES Message is then directly received by an SDR receiver, which acts as satellite terminal and MAMES Receiver (Alert Message decapsulation).

The Alert and MAMES message exchange between the MAMES Provider and the Alert Issuer (Interface I1) and Satellite MCC (Interface I2), respectively, relies on M2M Communication mechanisms based on Web Services paradigm.

In detail, the easy automation of the entire process of Alert Message provision and MAMES Message generation is obtained by the use of a high-level Python Web framework at the MAMES Provider and a python script at the Alert Issuer. As depicted in Figure 6 (Interface I1), as the Alert Issuer launches the script, the alert related information is submitted to the MAMES Alert Provider and the corresponding MAMES Message generation and storage in the local Data Base are triggered on the MAMES Provider side. The Satellite MCC periodically queries the MAMES Alert Provider for new MAMES Messages stored in the MAMES Provider Local Data Base (Interface I2). A python script is used by the Satellite MCC for managing the query and the MAMES Message download and formatting into a binary file for the consecutive upload to D-SAT.

It is worth highlighting that the D-SAT logical operations of the MAMES communication protocol and message validation, relies on the SatAlert Experiment task (SAE) and the Interface SatAlert Experiment task (ISAE), which are the two main functions of the on-board satellite operating system. The SAE logical functions include:

- Time validity check. Upon reception of a trigger command for transmission, the validity of the MAMES message previously stored in the transmission queue is verified (Case 1). 


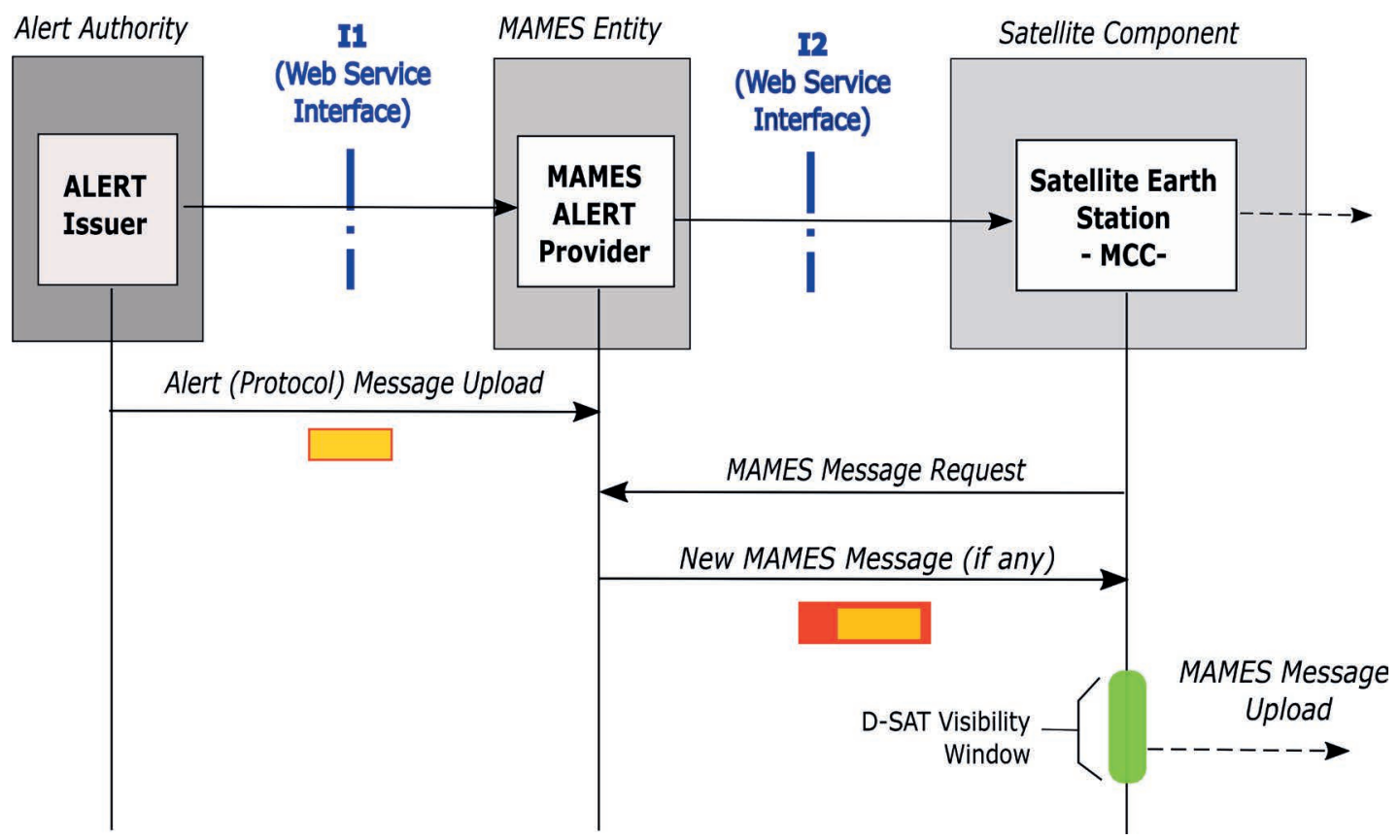

Figure 6.

M2M communication system: Web service interfaces.

- Time validity and priority checks. Upon reception of a new MAMES Message from the Satellite MCC, the SAE parses the received message to compare priority and validity of the new uploaded message with the previously stored message (Case 2).

The check results make the SAE decide for a message broadcasting or queue cancellation (Case 1 options) and for a message replacement or discarding (Case 2 options). The ISAE, which guarantees the SatAlert experiment independence from the rest of the mission by managing the software-hardware interaction between the transceiver and the on-board computer, is then informed about the SAE performed action.

In particular, the MAMES message upload by the Satellite MCC, the consecutive D-SAT on board processing and the storage (if required) in the On-Board Computer memory and the broadcast transmission activated by the MCC trigger command, are scheduled during the D-SAT available visibility windows (agreements on non-interference regulated by no. 4.4 of the ITU Radio Regulations). For each user on ground, three or four visibility windows of about $480 \mathrm{~s}$ per day (depending on the specific day) are provided. Figure 7 shows the satellite footprint on ground (yellow circle).

\section{D-SAT MISSIDN RESULTS}

\section{IN-LAB TESTS RESULTS}

After the D-SAT Qualification Model has successfully passed the tests campaign which was based on the Launch Service Provider (LSP) requirements, including mechanical (Sine and Random Vibration, Shock and Acceleration Test) and thermal-vacuum test, the D-SAT Flight Model (FM) integration and acceptance test campaign was completed. In April 2017, the D-SAT FM satellite delivered to the LSP, integrated within the P-POD deployer [30], and shipped to the PSLV's launch site in India.

An extended test campaign of Sat Alert operating functions (MAMES Message generation and on-board processing) has been performed with good results: the end-to-end communication between the Alert Issuer and Portable SDR user terminal was experimented in D-Orbit facility with all the real equipment, also emulating the path-loss attenuation using power attenuators in receiving chain.

\section{IN-QRBIT RESULTS}

D-SAT FM satellite was successfully launched on 23rd of June 2017 from the Satish Dhawan Space Centre, India, on-board the PSLV C-38 mission. Immediately since the first visibility window, the team was able to acquire the signal and receive the first telemetry. Launch and Early Operations Phase was completed within the first month of Mission and the functional tests proved that all satellite subsystems, including redundancies, are working properly. Focusing on the results of the alert messaging system, the satellite has successfully completed the SatAlert experiment. The whole chain from the alert message issue to the alert reception has been successfully tested and validated, demonstrating the proper functioning of the proposed system. All the building blocks have worked according to the specifications. In detail:

- MAMES message generation. Different types of MAMES messages have been generated (ALERT, CANCEL, UltraShort) and tested. The set of WS developed to let the main entities to interface one to each other and to automatically 


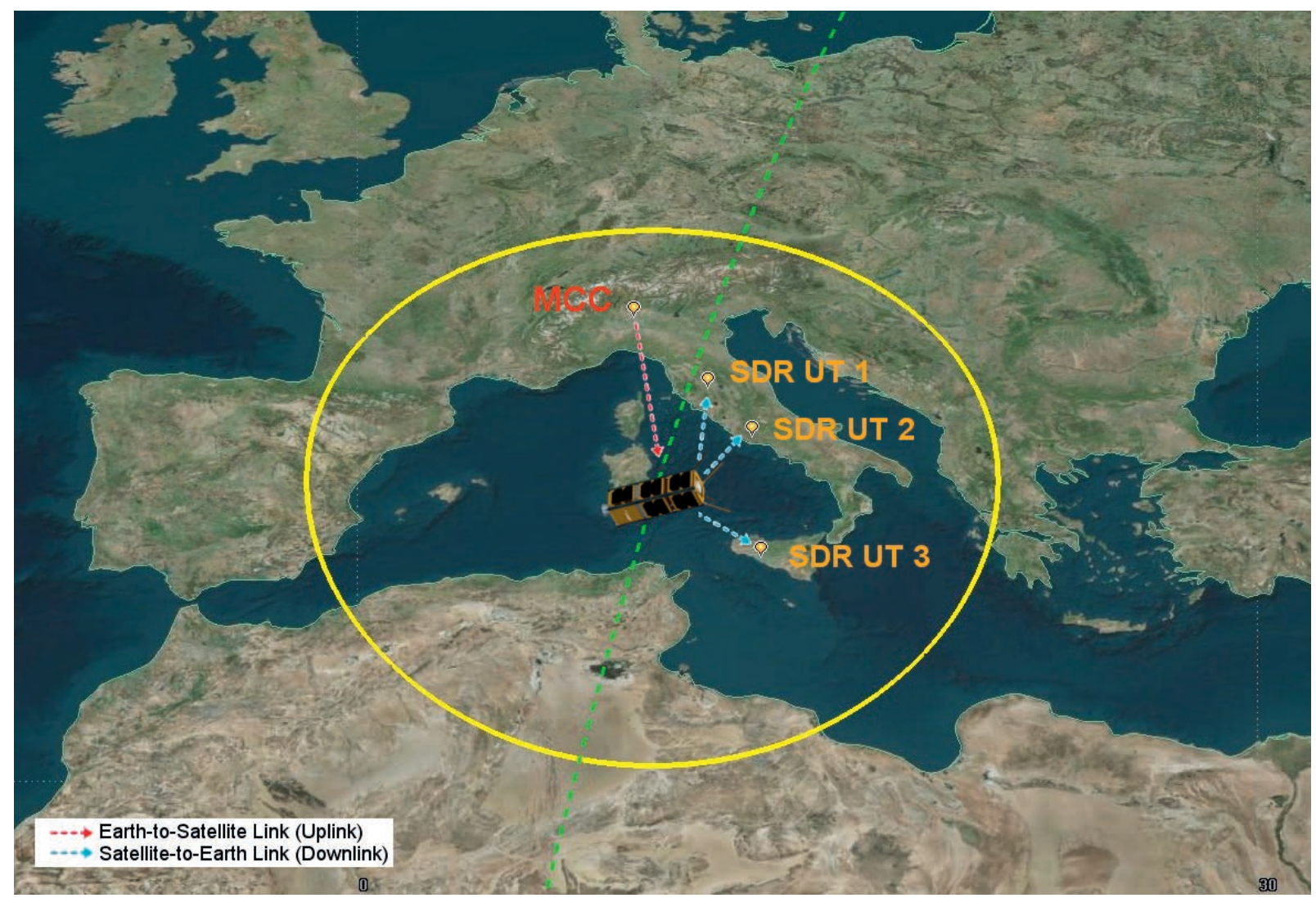

\section{Figure 7.}

D-SAT ground footprint.

activate the alert message encapsulation process (MAMES Messages generation) has worked properly and the Satellite MCC has been able to upload the generated messages.

- D-SAT on board processing and broadcasting. The D-SAT on board processing has allowed the priority and time validity checks making the SAE and ISAE act properly. The ALERT and UltraShort uploaded messages have been broadcasted based on their priority and time validity, while the CANCEL messages have allowed the on-board queue erase, if referring to the on-board stored message.

- SDR portable receiver. The low-complexity satellite SDRbased receiver has been able to correctly receive the MAMES messages directly by the D-SAT and decode them. The alert messages encapsulated into the MAMES payload have been successfully extracted. Both plain text and CAP alert messages have been tested.

The SatAlert experimental activity involved also European ham radio community that actively participated in the reception and extraction of the MAMES message transmitted by D-SAT, by using their own SDR receiver.

\section{CONCLUSIONS AND FUTURE WORK}

A flexible CubeSat-based system for data broadcasting has been proposed with the aim to meet the requirements of the advanced and multidisciplinary services, which relies on the integration of sensing, navigation, and communication capabilities. The highlevel design of the system architecture is provided together with some of potential application contexts which could benefit from the use CubeSat (Health and well-being; Smart, green and integrated transport; Security and emergency). Moreover, the applicability of the proposed system to an Emergency scenario has been shown and the experimental activity carried out in the framework of the D-SAT Mission has been presented as a proof of concept of the developed Alert Messaging System. The whole chain from the alert message issue to the alert reception has been tested and validated, considering: i) the alert message encapsulation process and the interfaces among the involved entities, on the transmission side; ii) the D-SAT on board processing and the consecutive broadcasting of MAMES messages; iii) the reception of them by a low complexity portable satellite receiver. The success of the SatAlert Experiment of the D-SAT Mission is presented through the analysis of the obtained results, demonstrating the effectiveness of the proposed system. All the building blocks, including the onboard software that parses and broadcasts the MAMES messages, worked according to the specifications, proving the feasibility of both the transmission over the space segment (LEO satellite) of alert messages encapsulated into MAMES protocol messages and their reception and decoding by a portable SDR UT.

Among the future developments of this activity, it is worth highlighting the definition of the suitable SmallSat constellations, which will permit a full deployment of the considered services by 
allowing a larger coverage and the analysis of the possible alternative scenarios: although the proposed system has been validated in an emergency context, future works may regard the validation in different application scenarios of the system and of the services which rely on the distribution of messages over a large area (e.g. traffic/transport information, advertising, etc.).

\section{ACKNOWLEDGMENTS}

This study is the result of a cooperation between CNIT (University of Florence) and D-Orbit. The D-SAT project was initially financed by D-Orbit investments and from December 2015 has received funding from the European Union's Horizon 2020 research and innovation programme under grant agreement No. 711193.

\section{REFERENCES}

[1] Del Re, E., Morosi, S., Ronga, L. S., and Jayousi, S. High capacity ground-, air- and space-based ICT networks for communications, navigation and sensing services in 2030. In CONASENSE-Role of ICT for Multi-Disciplinary Applications in 2030. River Publishers, 2016.

[2] Del Re, E., Morosi, S., Ronga, L. S., Jayousi, S., and Martinelli, A. Flexible heterogeneous satellite-based architecture for enhanced quality of life applications. IEEE Communications Magazine, Vol. 53, 5 (May 2015), 186-193, DOI: 10.1109/MCOM.2015.7105659.

[3] [Online] Available: https://magnitudespace.com/.

[4] [Online] Available: http://helioswire.com/.

[5] Villain, R. Facts \& figures on possible future directions of the SmallSat market. In Proceedings of the Final Proceedings at Small Satellites, System \& Services Symposium (4S), Valletta, Malta, 30 May-3 June 2016.

[6] Buscher, M., Funke, T., Patzphal, A., and Brie, K. Satellites might go viral - data proves it. In Proceedings of the Final Proceedings at Small Satellites, System \& Services Symposium (4S), Valletta, Malta, 30 May-3 June 2016.

[7] Crisp, N. H., Smith, K., and Hollingsworth, P. Launch and deployment of distributed small satellite systems. Acta Astronautica, Vol. 114, (2015), 65-78.

[8] Larsen, J. A., and Mortensen, H. P. In orbit validation of the AAUSAT3 SDR based AIS receiver. In Proceedings of the 2013 6th International Conference on Recent Advances in Space Technologies (RAST), Istanbul, Turkey, 2013, 487-491, DOI: 10.1109/RAST.2013.6581257.

[9] [Online] Available: http://www.outernet.is.

[10] [Online] Available: http://www.astrocast.net/.

[11] Ulversy, T. Software defined radio on digital communications: A new teaching tool. In Proceedings of the 2012 IEEE 13th Annual Wireless and Microwave Technology Conference (WAMICON), Vol. 12, 9781-46730128-2 (2012), 18.

[12] Maheshwarappa, M. R., and Bridges, C. P. Software defined radios for small satellites. In Proceedings of the 2014 NASA/ESA Conference on Adaptive Hardware and Systems (AHS), Leicester, England, 2014, 172-179, DOI: 10.1109/AHS.2014.6880174.

[13] [Online] Available: http://www.fleet.space/.

[14] [Online] Available: http://http//www.aistechspace.com/.
[15] Lofaldli, A., and Birkeland, R. Implementation of a software defined radio prototype ground station for CubeSats. In Proceedings of the Final Proceedings at Small Satellites, System \& Services Symposium (4S), Valletta, Malta, 30 May-3 June 2016.

[16] Maral, G., Bousquet, M., and Sun, Z. Satellite communications systems: Systems, techniques and technology. Wiley Series in Coтmunication and Distributed Systems, (5th ed.), ISBN: 9781119965091. Wiley, 2011.

[17] Fanfani, A., Morosi, S., Ronga, L. S., and Del Re, E. Frequency recovery techniques for TM/TC satellite modem in critical scenarios. Wiley International Journal of Satellite Communications and Networking, (2016), DOI: 10.1002/sat.1211.

[18] Mueller, K., and Muller, M. Timing recovery in digital synchronous data receivers. IEEE Transactions on Communications, Vol. 4, 5 (May 1976), 516-531, DOI: 10.1109/TCOM.1976.1093326, ISSN: 0090-6778.

[19] W3C. Web services architecture. [Online] Available: http://www. w3.org/TR/2004/NOTE-ws-arch-20040211/.

[20] Bray, T. The JavaScript object notation (JSON) data interchange format. (2014).

[21] Maynard, R. L. Chapter 6 health effects of urban pollution. In Air Quality in Urban Environments, Vol. 28, pp. 108-128. The Royal Society of Chemistry, 2009.

[22] Kampa, M., and Castanas, E. Human health effects of air pollution. In Proceedings of the 4th International Workshop on Biomonitoring of Atmospheric Pollution (With Emphasis on Trace Elements), Environmental Pollution, Vol. 151, no. 2, 2008, pp. 362-367.

[23] Chen, C.-M., Macwan, A., and Rupe, J. Network disaster recovery. IEEE Communications Magazine, Vol. 49, 1 (Jan. 2011), 26-27.

[24] Fanfani, A., Jayousi, S., Morosi, S., Ronga, L. S., and Del Re, E. Experimental low complexity CubeSat-based network for alert messages broadcasting. In Proceedings of the Global Wireless Summit 2016, Aarhus, Denmark, Nov. 24-27, 2016.

[25] Fanfani, A., Jayousi, S., Morosi, S., Ronga, L. S., Del Re, E., and Rossettini, L. Feasibility study of an alert messaging system by means of CubeSat, SDR and Web service technologies. In Proceedings of IEEE Globecom, Singapore, Dec. 2017.

[26] Toson, E., Fanfani, A., Morosi, S., Ronga, L. S., Martegani, P., and Rossettini, L. D-SAT mission: An in-orbit demonstration of a solid propellant satellite decommissioning device. In Proceedings of the Final Proceedings at Small Satellites, System \& Services Symposium (4S), Valletta, Malta, 30 May-3 June 2016.

[27] ETSI. Satellite earth stations and systems (SES); satellite emergency communications; multiple alert message encapsulation over satellite (MAMES). ETSI, Tech. Rep. TS 103 337, May 2015.

[28] ETSI. Satellite earth stations and systems (SES); satellite emergency communications (SatEC); multiple alert message encapsulation over satellite (MAMES) deployment guidelines. ETSI, Tech. Rep. TR 103 338, May 2015.

[29] Ronga, L. S., Jayousi, S., Pucci, R., Morosi, S., Berioli, M., Rammer, J., et al. Multiple alert message encapsulation protocol: Standardization and experimental activities. Palen, Bscher, Comes, and Hughes, Eds. In Proceedings of the ISCRAM 2015 Conference, Kristiansand, Norway, May 24-27, 2015.

[30] The CubeSat Program. CubeSat design specification, Rev. 13. Cal Poly SLO. 